\title{
Controlling Visible Light Driven Photoconductivity in Self-Assembled Perylene Bisimide Structures
}

\author{
James J. Walsh, ${ }^{\dagger,}$ Jonathan R. Lee, ${ }^{\ddagger}, \S$ Emily R. Draper, ${ }^{\dagger}$ Stephen M. King, $"$ Frank Jäckel, ${ }^{\ddagger, \S}$ \\ Martijn A. Zwijnenburg, ${ }^{\perp}$ Dave J. Adams, ${ }^{*}{ }^{\dagger}$ and Alexander J. Cowan ${ }^{*, \dagger, *}$ \\ ${ }^{\dagger}$ Department of Chemistry, University of Liverpool, Crown Street, Liverpool L69 7ZD, United Kingdom. \\ ${ }^{\ddagger}$ Stephenson Institute for Renewable Energy, University of Liverpool, Peach Street, Liverpool L69 7ZF, United Kingdom \\ ${ }^{\S}$ Department of Physics, University of Liverpool, Oxford Street, Liverpool L69 7ZE, United Kingdom \\ "ISIS Facility, Rutherford Appleton Laboratory, Science and Technology Facilities Council, Didcot, Oxfordshire OX11 0QX, United \\ Kingdom \\ ${ }^{\perp}$ Department of Chemistry, University College London, 20 Gordon Street, London WC1H 0AJ, United Kingdom
}

\section{Supporting Information}

\begin{abstract}
Alanine-functionalized perylene bisimides (PBIA) are promising photoconductive materials. PBI-A selfassembles at high concentrations ( $\mathrm{mM}$ ) into highly ordered wormlike structures that are suitable for charge transport. However, we previously reported that the photoconductive properties of dried films of PBI-A did not correlate with the electronic absorption spectra as activity was only observed under UV light. Using transient absorption spectroscopy, we now demonstrate that charge separation can occur within these PBI-A structures in water under visible light. The lack of charge separation in the films is shown by DFT calculations to be due to a large ion-pair energy in the dried samples which is due to both the low dielectric environment and the change in the site of hole-localization upon drying. However, visible light photoconductivity can be induced in dried PBI-A films through the addition of methanol vapor, a suitable electron donor. The extension of PBI-A film activity into the visible region demonstrates that this class of self-assembled PBI-A structures may be of use in a heterojunction system when coupled to a suitable electron donor.
\end{abstract}

\section{INTRODUCTION}

Perlyene bisimides (PBIs) are increasingly being explored for use in organic photovoltaic ${ }^{1}$ and solar fuel applications ${ }^{2}$ due to their large visible extinction coefficients, high chemical and thermal stabilities, and potential synthetic tunability. ${ }^{3}$ PBIs are known to self-assemble through $\pi-\pi$-stacking even at very low $\left(<10^{-5} \mathrm{M}\right)$ concentrations leading to the formation of aggregates, the structure of which can greatly impact upon the photophysical and photochemical properties of the PBI. However, stacking and subsequent supramolecular structure formation can be difficult to control. One promising approach to controlling aggregation is to develop materials that are able to self-assemble into well-defined structures. We, among others, have recently reported on a class of amino acid appended PBI structures that can self-assemble in solution to form supramolecular wormlike micelles which persist on the microns distance scale. ${ }^{4-6}$ The structures consist of slip-stack helical bundles of $\pi$-stacked $H$-aggregated monomers with the $\pi-\pi$ interactions offering a feasible route for exciton diffusion and long-range charge transport. ${ }^{4,7}$ Such PBI supramolecular structures have also been shown to be n-type photoconductors with conductivity being due to the formation of a $\mathrm{PBI}^{\bullet-}$ state. $^{8-10}$ Often, such materials are highly sensitive to the presence of quencher molecules such as $\mathrm{O}_{2}$; however, the photoinduced conductivity of an alanine functionalized PBI (PBI-A, structure Figure 1a) film was found to persist for many hours even in air in the dark, ${ }^{6}$ making them potentially interesting alternative electron acceptors in organic photovoltaic cells. Although this was a promising result, the photoresponse of dried PBI-A films did not correlate well with the measured UV/vis absorption spectrum, with photoconductivity only occurring upon excitation into the $S_{0}-S_{2}$ transition using UV light (ca. $365 \mathrm{~nm}$ ). Excitation into the strongly absorbing $\mathrm{S}_{0}-\mathrm{S}_{1}$ transition resulted in no measurable photoconductivity. ${ }^{6}$ Further analysis of other PBI-derived structures in the literature indicates that a similar loss of visible light activity may also be occurring in other related amino acid appended PBI photoconductive and photocatalytic samples. ${ }^{2,5}$ If visible light photoconductivity could be coupled to the known oxygen tolerance of these self-assembled structures, it

Received: June 20, 2016

Revised: August 1, 2016

Published: August 1, 2016 

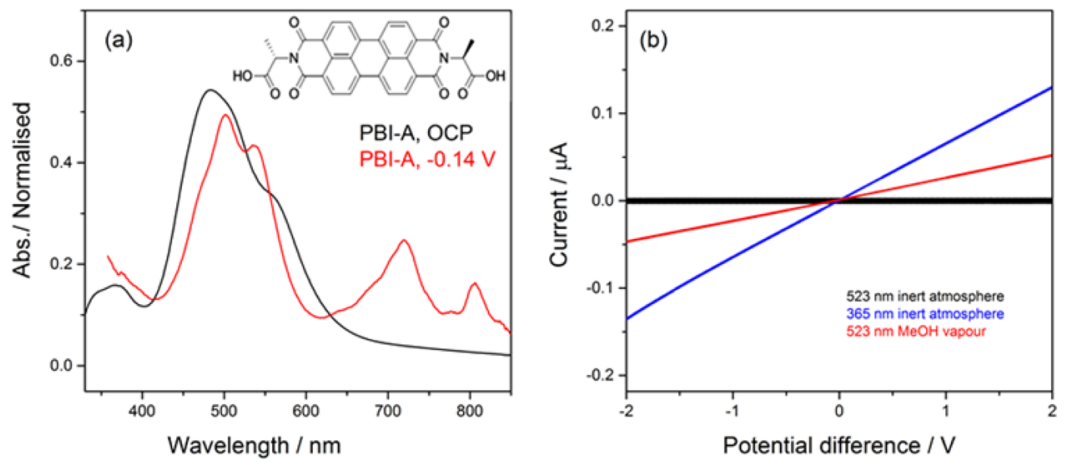

Figure 1. (a) UV/vis spectroelectrochemistry of PBI-A structures dried as a film on FTO at open circuit potential (black) and following the first reduction (ca. $-0.14 \mathrm{~V}$ versus a $\mathrm{Ag}$ wire pseudo-reference electrode, red) in $\mathrm{CH}_{3} \mathrm{CN}, 0.1 \mathrm{M} \mathrm{TBA} \mathrm{PF}_{6}$. (b) $I-V$ curves for a dried solution of PBI-A under controlled atmospheres under illumination at the wavelengths indicated. The monomer structure of PBI-A is shown in the inset of (a).

would represent an important breakthrough in the search for alternative acceptor materials for organic photovoltaics and novel organic photocatalysts.

Transient absorption (TA) spectroscopy has been used extensively to study PBI donor-acceptor systems, providing important insights into the formation and lifetimes of charge separated and excited states. ${ }^{11,12}$ In addition, exciton ${ }^{13}$ and charge transport $^{14}$ efficiencies have been measured. Charge separation occurs in oligomeric self-assembled structures and has been shown to be accelerated in closely bound aggregates, with numerous reports of visible light driven charge separation in PBIs in solution. ${ }^{15,16}$ Here, we present a mechanistic study that explores the factors controlling charge separation both in solution and as a dried film and hence the spectral response of PBI-A. We also demonstrate the viability of the use of these materials in a potential OPV heterojunction, as in the presence of a suitable donor molecule where visible light driven photoconductivity occurs.

\section{EXPERIMENTAL METHODS}

Materials. PBI-alanine (PBI-A, $\left[N, N^{\prime}\right.$-di(L-alanine)-perylene-3,4:9,10-tetracarboxylic acid bisimide]) was synthesized as previously described. ${ }^{6}$ PBI-A solutions were prepared at a concentration of $25 \mathrm{mg} / \mathrm{mL}(47 \mathrm{mM})$ using an equimolar quantity of dilute aqueous $\mathrm{NaOH}(0.1 \mathrm{M})$ to dissolve the solid and then made up to $25 \mathrm{mg} / \mathrm{mL}$ with distilled water. Solutions were left stirring overnight to ensure all the solid had dissolved. To make films, $25 \mu \mathrm{L}$ of PBI-A solution were drop-cast onto either a glass microscope slide using a $5 \times 5 \mathrm{~mm}$ mask or a precleaned fluorine tin oxide electrode (FTO, TEC-15, Pilkington). Tetrabutylammonium hexafluorophosphate (TBA $\mathrm{PF}_{6}$, Sigma-Aldrich) was recrystallized from ethanol prior to use. Acetonitrile $\left(\mathrm{CH}_{3} \mathrm{CN}, \mathrm{VWR}\right.$, spectroscopic grade) and methanol ( $\mathrm{MeOH}$, Sigma, HPLC grade) were used as received. Milli-Q water was used throughout.

Steady-State Spectroscopy. UV/vis absorption data were obtained using a Shimadzu $2550 \mathrm{UV} /$ vis spectrophotometer using cells of various path lengths suitable to the range of concentrations studied. Spectroelectrochemistry (SEC) was performed on films of PBI-A cast onto FTO as the working electrode. The films were doctor-bladed and allowed to dry in air in the dark; the edges of the film were then sealed with epoxy resin (Loctite Hysol $3422 \mathrm{~A}+\mathrm{B}$ ) and allowed to dry overnight in the dark. SEC was performed in a quartz cell using $0.1 \mathrm{M} \mathrm{TBA} \mathrm{PF}_{6}$ in argon-purged acetonitrile as the supporting electrolyte, a Pt wire as the counter electrode, and a Ag wire as the pseudoreference electrode. A PalmSens ${ }^{3}$ potentiostat was used for SEC measurements. Fluorescence spectra were collected using a PerkinElmer Fluorescence Spectrometer LS55 using cells of various path lengths suitable to the range of concentrations studied.

Photoconductivity Measurements. Photoconductivity was measured using a two-electrode measurement. Films were prepared on microscope slides between two silver electrodes spaced $3 \mathrm{~mm}$ apart, with a contact to a copper wire being made using silver paste (RS Electronics). Prior to use, the film was dried in air overnight, and then epoxy resin was placed over the silver electrodes, which was again left to dry overnight. Samples were then sealed in a $1 \times 2 \mathrm{~cm}$ quartz cuvette with a B24 adapter (Starna) and septum through which the wires could pass and be either left in air, purged with argon, or purged with argon presaturated with $\mathrm{MeOH}$ vapor as described in the text. A 365 or 523 nm LED (LED Engin) powered by a TTi QL564P power supply was used, and the incident light intensity on the sample was $1 \mathrm{~mW} / \mathrm{cm}^{2}$. A PalmSens ${ }^{3}$ potentiostat was used for all photoconductivity measurements employing linear sweep voltammetry from -2 to $+2 \mathrm{~V}$ at $50 \mathrm{mV} / \mathrm{s}$.

Femtosecond and Microsecond Transient Absorption (fsTA and $\mu$ sTA) Spectroscopies. Femtosecond TA spectroscopy was carried out using a PHAROS laser (Light Conversion, Ltd.) operating at $10 \mathrm{kHz}$ coupled to an ORPHEUS optical parametric amplifier (Light Conversion, Ltd.) in tandem with a LYRA harmonic generator (Light Conversion, Ltd.) to produce the desired wavelength for sample excitation. The pump beam intensity was adjusted with a neutral density filter so as to achieve approximately equal photon fluxes at different wavelengths. Typical pulse energies were on the order of $10 \mathrm{~nJ}$. The pump wavelength was tuned to either UV $(365 \mathrm{~nm})$ or visible $(490 \mathrm{~nm})$ as needed. In contrast to the $523 \mathrm{~nm}$ LED used for photoconductivity measurements (see above), $490 \mathrm{~nm}$ was chosen as the visible pump wavelength to remove laser scatter in a region of spectral interest. A portion of the PHAROS output was also split off to pump a sapphire crystal to generate a white light continuum for the probe beam, which provided for spectral observation in the region $450-900 \mathrm{~nm}$. The probe beam was focused to a spot size of $\sim 100 \mu \mathrm{m}$ diameter on the sample and was overlapped completely by the pump beam. Spectra were acquired with a HELIOS transient absorption spectrometer (Ultrafast Systems, LLC). The time resolution of the setup is $\sim 400$ fs. Measurements were performed by randomly stepping the 

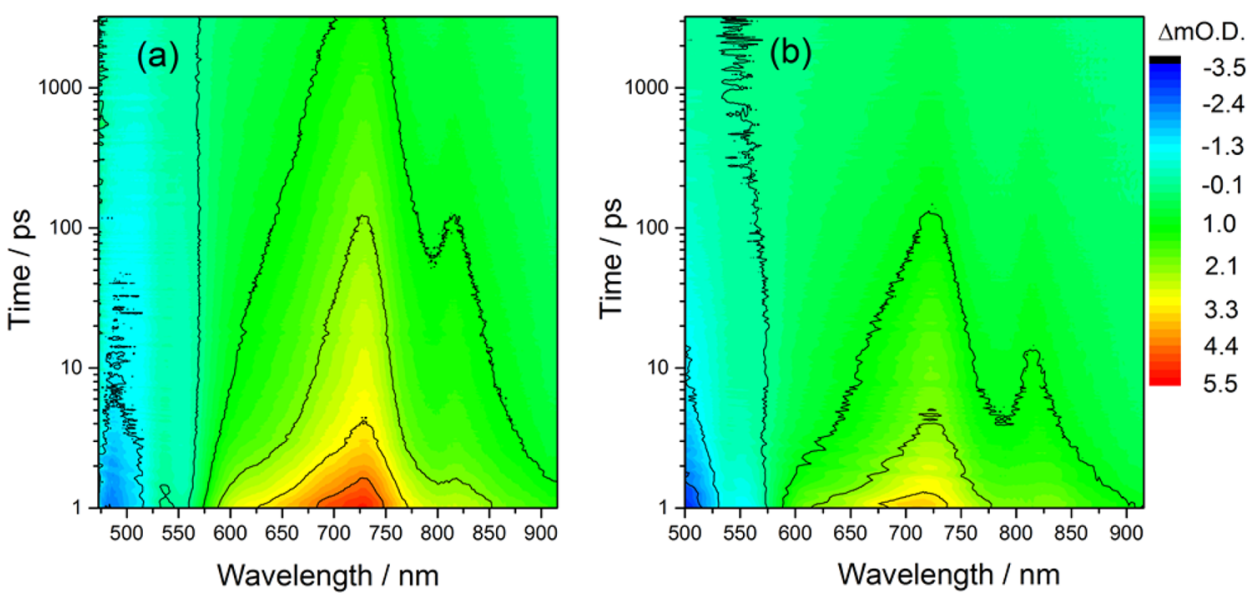

Figure 2. (a) TA spectra of PBI-A in water ( $\mathrm{pH} 9)$ at $47 \mathrm{mM}$ following $\mathrm{UV}(10 \mathrm{kHz}, 365 \mathrm{~nm}, 100 \mu \mathrm{W})$ or (b) visible light excitation (10 kHz, 490 $\mathrm{nm}, 470 \mu \mathrm{W})$.

optical delay line and averaging for $1 \mathrm{~s}$ at each delay time. Five consecutive scans were collected and aggregated to produce each spectrum. Solution samples were measured in a $2 \mathrm{~mm}$ path length quartz cuvette or between two glass slides. The solution was stirred rapidly throughout acquisition. Thin film samples were formed upon drying of a $25 \mu \mathrm{L}$ drop of aqueous stock PBI-A solution $(47 \mathrm{mM})$. Spectra of thin film samples were recorded sealed in a quartz cuvette purged with argon or argon presaturated with $\mathrm{MeOH}$ vapor. Data were chirp corrected using the Surface Xplorer software package. Microsecond ( $1 \mu \mathrm{s}-1 \mathrm{~s})$ TA spectroscopy measurements were carried out on the same samples using a system described elsewhere that is based on a Nd:YAG laser (Continuum Surelite) and OPO (Continuum SLOPO-1). ${ }^{17}$

Density Functional Calculations. All calculations were performed using the B3LYP ${ }^{18,19}$ functional with the D3 empirical dispersion correction ${ }^{20}$ and the $\mathrm{DZP}^{21}$ or def2$\mathrm{TZVP}^{22}$ basis set. Calculations on the neutral and deprotonated PBI-A additionally employed the COSMO continuum solvation model $^{23}$ with a relative dielectric permittivity value of 80 to model the dielectric screening effect of water. Frequency calculations were performed on the different stationary points obtained by geometry optimization to verify that they were indeed minima. Finally, all ground state calculations were performed using the Turbomole 6.6 code. $^{24,25}$

\section{RESULTS AND DISCUSSION}

The UV/vis spectrum of the as-cast film of PBI-A is shown in Figure 1a. The spectrum is dominated by the $S_{0}-S_{1}$ electronic transition, composed of a series of closely spaced vibronic modes (vibronic states given in brackets). Spectral fitting (Figure S1) reveals the presence of three transitions at $566(0-$ $\left.0^{\prime}\right), 504\left(0-1^{\prime}\right)$, and $467\left(0-2^{\prime}\right) \mathrm{nm}$, with the greatest contribution from the $\left(0-1^{\prime}\right)$ mode, indicating extensive $\mathrm{H}$ aggregation and cofacial interactions, which will aid the formation of conductive pathways. ${ }^{12}$ Comparison with the $\mathrm{UV} / \mathrm{vis}$ spectrum of PBI-A at low concentration $\left(10^{-7} \mathrm{M}\right)$ indicates that a high level of aggregation between the PBI units occurs at $47 \mathrm{mM}$ (Figure S2). ${ }^{3}$ The $\mathrm{S}_{0}-\mathrm{S}_{2}$ transition is also apparent for the PBI-A film in the UV with a maximum at ca. $360 \mathrm{~nm}$. Previous structural analyses have demonstrated the PBI aggregates in the dried films are in the form of long onedimensional wormlike micelle structures. ${ }^{6,26}$ In line with our past study, excitation into the $S_{0}-S_{1}$ transition (523 nm LED) of the PBI-A film under an argon atmosphere is seen to lead to negligible change in the electrical resistance of the sample (Figure $1 \mathrm{~b}$ ), while excitation of the $S_{0}-S_{2}$ transition $(365 \mathrm{~nm}$ ) leads to a large decrease in resistance with the sample showing an Ohmic response indicating that the samples are photoconductive under UV light. ${ }^{6}$ This result confirms that longrange charge transport through the extended $\pi-\pi$ stacked structure of the dried PBI-A film is possible and that the lack of photoconductivity following $S_{0}-S_{1}$ excitation may be due to a lack of initial charge separation or the presence of only shortlived charge-separated states.

In order to examine the potential roles that supramolecular structure and solvent have in controlling charge separation, we initially studied the self-assembled PBI-A structures in solution. It is well understood that the nature of the aggregates formed in PBI solutions is highly dependent upon the PBI concentration. ${ }^{3}$ $\mathrm{UV} /$ vis and emission spectroscopy indicate that the PBI-A concentration has to be on the order of $1 \times 10^{-7} \mathrm{M}$ for monomer like behavior to be observed (Figure S2a). In aqueous solutions at $47 \mathrm{mM}$ ( $\mathrm{pH}$ 9), the concentration used for preparing the PBI-A films, both UV/vis and fluorescence measurements indicate that significant PBI-A aggregation has occurred evidenced by the quenching of the monomer type fluorescence fine structure at 546 and $583 \mathrm{~nm}$ (Figure S2b), the appearance of a weak fluorescence at $652 \mathrm{~nm}$ (Figure S2b, S2c), and the increase in the contribution of the $\left(0-1^{\prime}\right)$ transition of the UV/vis spectrum. ${ }^{5}$ Spectroelectrochemistry (SEC) of the reduced film is also shown in Figure $1 \mathrm{a}$ and is typical of a reduced PBI species. ${ }^{6}$ Viscosity measurements of the solution indicate the presence of large-scale aggregates (Figure S4), attributed to wormlike micelles, in agreement with the previous analysis of the dried sample by SEM and XRD which identified that the facially stacked aggregates had a PBI separation of ca. $3.5 \AA$ with structures on the micron scale. ${ }^{6}$ Further confirmation of the presence of similar structures in both the solution studies and the dried films is achieved using small angle neutron scattering (SANS). The scattering from the solution sample could be fitted to a wormlike chain model (as the Kratky-Porod flexible cylinder model in the SasView software package (https://www.sasview.org, Figure S5). We have successfully used this model for other related systems, ${ }^{27,28,20}$ The fit suggests that the system contains structures with a radius of $6.4 \pm 0.1 \mathrm{~nm}$, with a Kuhn length of $10.1 \pm 1.3 \mathrm{~nm}$. 
The TA spectra of PBI-A solutions at $47 \mathrm{mM}$ using both UV $(365 \mathrm{~nm})$ and visible $(490 \mathrm{~nm})$ excitation indicate that the excited state species present after 1 ps are very similar, showing a loss of the aggregated ground state $(504, \mathrm{ca} .560 \mathrm{~nm})$ and a long-lived broad positive band at $725 \mathrm{~nm}$ with shoulders at ca. 540, ca. 625, and $818 \mathrm{~nm}$ (Figures 2, 3). The newly formed

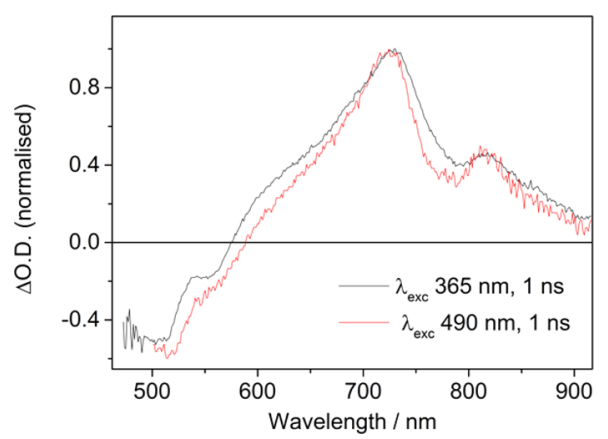

Figure 3. TA spectrum of PBI-A in water $(\mathrm{pH} 9)$ at $47 \mathrm{mM}$ showing the presence of the same transient species $1 \mathrm{~ns}$ after excitation following initial excitation of either the $S_{0}-S_{2}(365 \mathrm{~nm})$ or $S_{0}-S_{1}(490$ $\mathrm{nm})$ transitions.

intermediate species under both UV and visible light excitation correlates well with both the previously reported spectrum of the $\mathrm{PBI}^{\circ-}$-A in solution ${ }^{6}$ and of the electrochemically generated $\mathrm{PBI}^{\circ-}$-A in a dried film $(541,723,806, \mathrm{ca} .635 \mathrm{~nm}$ (sh), Figure $1 b)$ indicating that excitation of either the $S_{0}-S_{1}$ or $S_{0}-S_{2}$ transition leads to the formation of a charge-separated state with the electron localized on the PBI core. The broadness of the PBI radical feature may indicate a degree of charge migration within the aggregate with previous studies noting a broadening of spectral features in aggregated PBIs. ${ }^{11,29}$

The reduced PBI and the PBI-A ground state bleach are observed to partially recover at the same rate (Figures S6, S7) with kinetics that can be fitted to a minimum of three exponential components $\left(\tau_{1}=\mathrm{ca} .1 \mathrm{ps}, \tau_{2}=17( \pm 0.9)\right.$ ps and $\tau_{3}$ $=257( \pm 16)$ ps at ca. $820 \mathrm{~nm}, 490 \mathrm{~nm}$ excitation $)$. A singular value decomposition analysis did not identify any additional spectral features beyond that of the PBI-A ground state and the reduced PBI, and it is proposed that the triexponential fit represents a parametrization of the dispersive kinetics, indicating that multiple recombination pathways exist in the supramolecular structure. It is also notable that a significant (ca. $10 \%$ ) concentration of the photogenerated reduced PBI persists beyond $3.2 \mathrm{~ns}$. The formation of such a long-lived radical anion also supports the hypothesized anion migration through the PBI structure. Alternatively, the lack of charge recombination may indicate that the localization of the cation is preventing charge carrier recombination. It is therefore important that the nature of the electron donor site is identified.

Based upon the oxidation $\left(E_{\mathrm{OX}}=0.80 \quad \mathrm{~V}_{\mathrm{Ag} / \mathrm{AgCl}}\right)$ and reduction $\left(E_{\mathrm{RED}}=-0.56 \mathrm{~V}_{\mathrm{Ag} / \mathrm{AgCl}}\right)$ potentials of PBI-A at ca. $\mathrm{pH} 9$ measured by square-wave voltammetry (Figure S8), and the reported oxidation potentials of alanine (ca. 0.4-0.65 $\left.\mathrm{V}_{\mathrm{Ag} / \mathrm{AgCl}}\right)^{22,30}$ in basic solution and the PBI core of similar materials (ca. 1.3-1.5 V), ${ }^{31,32}$ it is proposed that the amino acid substituent can act as an electron donor, with the PBI core being the acceptor unit following photoexcitation of the solution. Furthermore, we observe no spectral features that are readily assignable to a PBI radical cation in Figure 2, supporting our assignment of the alanine unit as the electron donor. ${ }^{32,33}$ As the spectrum of oxidized alanine is expected to be outside the spectral window of this study, ${ }^{34}$ we are unable to ascertain how the oxidized alanine group evolves with time. Therefore, we assign the photochemically generated transient with features at 540 , ca. 625 , and $818 \mathrm{~nm}$ to $\mathrm{PBI}^{\bullet-} \mathrm{A}^{\prime}$ (where $\mathrm{A}^{\prime}$ is an alanine residue following initial oxidation of undefined state).

To further aid the spectral assignment, DFT calculations have been carried out to study the excess electron and hole localization and thus radical anion and cation formation, as well as to predict the PBI-A reduction potentials. Initially, we examined a neutral, a singly deprotonated, and a doubly deprotonated PBI-A molecule in water, Table S1. In the case of neutral PBI-A in water, an excess electron and hole are both predicted to localize on the PBI core of PBI-A. However, at the experimental $\mathrm{pH}$ studied $(\mathrm{pH} 9)$, the carboxylic acid of the alanine group is expected to be deprotonated. Calculations show that deprotonation leads to localization of the hole on the deprotonated alanine group(s), while the radical anion is still predicted to involve localization of the excess electron on the PBI core. This is all in-line with predictions of the redox potentials of free alanine and unsubstituted PBI, given in Table S1, which show an excellent correlation to the measured redox potentials, quoted above. The oxidation potential of neutral free alanine in water $(6.18 \mathrm{~V}$ vs. vacuum and $1.62 \mathrm{vs} . \mathrm{Ag} / \mathrm{AgCl})$ is predicted to lie significantly more positive than that of unsubstituted PBI (5.75 vs. vacuum and 1.20 vs. $\mathrm{Ag} / \mathrm{AgCl}$ ) and thus is harder to oxidize. Deprotonated free alanine in contrast has a significantly smaller oxidation potential (4.80 vs. vacuum and 0.31 vs. $\mathrm{Ag} / \mathrm{AgCl}$ ) and is thus easier to oxidize than unsubstituted PBI. This different localization of excess electron and hole could potentially have a positive effect on suppressing electron-hole recombination by moving them onto different "sublattices" and may be a further contributing factor behind the long-lived $\mathrm{PBI}^{\bullet-}$ state. DFT calculations also allow us to predict the energy required to make a pair of charge-separated states, i.e., a radical cation and radical anion separated by a large distance, commonly referred to as the quasiparticle or transport gap in extended solids but below discussed as the ion pair energy. The energy required to generate a pair of charge-separated states, radical ions, for a deprotonated PBI-A in water is calculated to be of the order of $2 \mathrm{eV}$ (see Table S2). In practice, stacking in solids or supramolecular aggregates will further slightly reduce this value (and that calculated for $\mathrm{Na}_{2}$ PBI-A below) by several hundred $\mathrm{meV}$ as a result of hybridization of the frontier orbitals of the different molecules; nonetheless, as shown below, the calculations agree well with the observed experimental behavior. This relatively small (ca. $2 \mathrm{eV}$ ) ion pair energy for deprotonated PBI-A in solution means visible light excitation $(528 \mathrm{~nm}, 2.35 \mathrm{eV})$ into the $S_{0}-S_{1}$ transition should be able to photoionize the excited state into charge-separated states. Experimentally, the Gibbs energy of photoinduced electron transfer in a donor-acceptor system ${ }^{35,36}$ can also be estimated using eq 1, which takes into account the donor and acceptor electrochemical redox potentials, the Coulombic interactions (C) between the two ions, and their the difference in solvation energies between the system of interest and the solvent that $E_{\mathrm{OX}}$ and $E_{\mathrm{RED}}$ were measured in $(S)$. Indeed, in water at $\mathrm{pH} 9$ we estimate an ion pair energy of ca. $1.3 \mathrm{eV}$, and we calculate that a large driving force exists for charge transfer from the lowest energy singlet excited state $\left(\Delta G_{\mathrm{ET}} \sim-1 \mathrm{eV}\right.$, see Table S3 for full details), in agreement with the TA observations of 

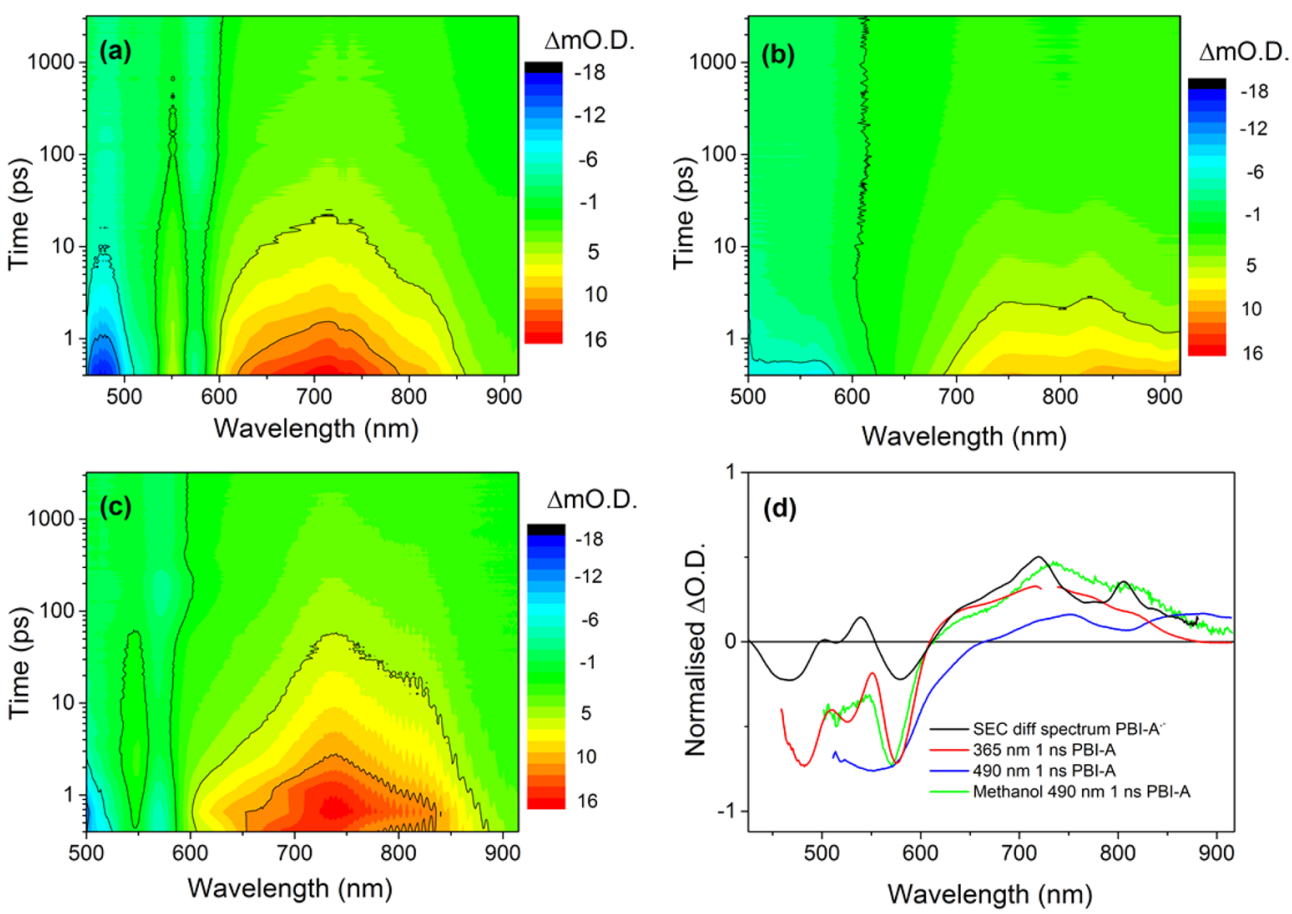

Figure 4. (a) TA spectra of a PBI-A film under an argon atmosphere following excitation of the $\mathrm{S}_{0}-\mathrm{S}_{2}(10 \mathrm{kHz}, 365 \mathrm{~nm}, 100 \mu \mathrm{W})$ or $(\mathrm{b}) \mathrm{S}_{0}-\mathrm{S}_{1}$ transitions $(10 \mathrm{kHz}, 490 \mathrm{~nm}, 470 \mu \mathrm{W})$ show the formation of two different transient states on the picosecond-nanosecond time scale. (c) Addition of methanol vapor enables the formation of a $\mathrm{PBI}^{\bullet-}-\mathrm{A}^{\prime}$ within the PBI-A film following $490 \mathrm{~nm}$ excitation. (d) Comparison of the TA spectra at $1 \mathrm{~ns}$ recorded under the conditions described in $(\mathrm{a}-\mathrm{c})$ and the electrochemically generated spectrum of $\mathrm{PBI}^{\bullet-}-\mathrm{A}$ in a $\mathrm{PBI}$ film at $-0.14 \mathrm{~V}$ vs $\mathrm{Ag} / \mathrm{Ag}^{+}$.

efficient charge separation and the DFT calculations outlined above.

$$
\Delta G_{\mathrm{ET}}=E_{\mathrm{OX}}-E_{\mathrm{RED}}-E^{*}-C+S
$$

It is therefore apparent that the visible light excitation of PBIA supramolecular structures in solution, which are similar to those employed in dried films, can lead to the formation of long-lived separated charges. TA studies on solid PBI-A films prepared by drying the $47 \mathrm{mM} \mathrm{pH} 9$ solutions on glass slides are now presented to ascertain if the dielectric environment is controlling the activity, Figure 4. Following excitation of the sample at $365 \mathrm{~nm}\left(\mathrm{~S}_{0}-\mathrm{S}_{2}\right.$ transition), we observe the ground state bands being bleached and the formation of a new transient species at ca. 550, ca. 725, and $810 \mathrm{~nm}$, Figure 4a), which shows excellent agreement with both the electrochemically generated spectrum of a $\mathrm{PBI}^{\bullet-}$-A radical in a dried film (Figure 4d) and the transient spectrum recorded for the same system in aqueous solution at $\mathrm{pH} 9$ (Figure 2a) allowing us to assign the transient species in the solid film to the charge-separated $\mathrm{PBI}^{\bullet-}-\mathrm{A}^{\prime}$ state. We find the $\mathrm{PBI}^{\circ-}-\mathrm{A}^{\prime}$ formed under UV excitation in the dried film decays with kinetics that can be fitted to a minimum of three exponential components again indicating that multiple recombination pathways exist in the supramolecular structure $\left(\tau_{1}=\right.$ ca. $1 \mathrm{ps}, \tau_{2}=15.9( \pm 0.5)$ and $\left.\tau_{3}=356( \pm 26) \mathrm{ps}\right)$ and approximately $>10 \%$ of the initially formed $\mathrm{PBI}^{\circ}{ }^{-}-\mathrm{A}^{\prime}$ is present $3.2 \mathrm{~ns}$ after excitation (Figure S9). We can also detect the $\mathrm{PBI}^{\bullet-}{ }^{-} \mathrm{A}^{\prime}$ species on the millisecond time scale following pulsed laser excitation (Figure S10). It is this population of long-lived charge PBI based radicals that are responsible for the photoconductivity under UV irradiation. ${ }^{5,6}$ In contrast, following excitation of the PBI-A film with visible $(490 \mathrm{~nm}$, $\mathrm{S}_{0}-\mathrm{S}_{2}$ ) light we observe a transient spectrum with maxima at ca. 740 and $830 \mathrm{~nm}$ at 1 ps (Figure 4b), markedly different to that recorded for PBI-A in solution at an equivalent concentration. We observe no evidence for fast charge separation and the generation of a $\mathrm{PBI}^{\circ-}-\mathrm{A}^{\prime}$ species (Figure $4 \mathrm{~d})$, in line with the lack of photoconductivity under visible light. ${ }^{6}$ The excited state with maxima at 740 and $830 \mathrm{~nm}$ is tentatively attributed to the formation of ${ }^{1} * \mathrm{PBI}\left(\tau_{1}<1 \mathrm{ps}, \tau_{2}=\right.$ $7.1( \pm 0.2)$ ps and $\tau_{3}=107( \pm 5.4)$ ps, at $\left.830 \mathrm{~nm}\right)$ which rapidly relaxes back to the ground state leaving only a minimal residual bleach $(<5 \%)$ at the longest time scales studied, Figure S11.

The lack of formation of the charge-separated state in solid films may be rationalized through consideration of the effective dielectric constant of the dried PBI-A (film, $\varepsilon \sim 3$ ) compared to the solvent dielectric for the solvated PBI-A structures (water, $\varepsilon$ $\sim 80$ ). Environments with a high relative dielectric permittivity, such as aqueous solutions, screen the free charge of the excess electron and hole and renormalize (reduce) ion pair energies/ quasiparticle gap, and multiple examples exist for PBI materials where charge separation can be controlled by the solvent environment. ${ }^{35,36}$ Experimentally we can estimate the effect of changing the dielectric environment through the use of eq 1 , providing it is assumed that the site of hole and electron localization remains the same. In this case, we estimate a decrease in the driving force for charge separation in the film compared to in solution by ca. $0.7 \mathrm{eV}$ (Table S3). However, while reductive spectroelectrochemistry (Figure $4 \mathrm{~d}$ ) indicates that the electron on the PBI-A film still resides on the PBI core, experiments to identify the site of hole localization on the dried PBI-A film were unsuccessful due to the insulating nature of the nonreduced PBI film. Importantly, DFT calculations on model systems indicate that the site of hole localization in solution and in the solid may be significantly different. As the dried films are prepared from a $\mathrm{NaOH}$ ( $\mathrm{pH}$ 9) PBI solution, we examined two versions of a Na-substituted PBI-A: a single $\mathrm{Na}_{2} \mathrm{PBI}-\mathrm{A}$ molecule in the gas phase and a $\mathrm{Na}_{2} \mathrm{PBI}-\mathrm{A}$ molecule in the gas phase with two additional coordinated water molecules per sodium cation, 
i.e., the simplest possible models of fully and partially dried down $\mathrm{Na}_{2}$ PBI-A solutions. Focusing on the radical cation, the hole is predicted in both of these systems to localize for approximately $50 \%$ on the two alanine groups and for approximately $50 \%$ on the PBI core. In contrast, the excess electron of the radical anion localizes exclusively on the PBI core for both models. Another noticeable difference between $\mathrm{Na}_{2} \mathrm{PBI}-\mathrm{A}$ and (de)protonated PBI-A is that when taking nuclear relaxation into account in the latter the hole typically localizes on one of the two alanine groups while in the case of $\mathrm{Na}_{2}$ PBI-A no such symmetry breaking is predicted to occur. Crucially, our calculations indicate that for our model system representing the low dielectric environment of the dried film the ion pair energy has increased from 2 to over $4 \mathrm{eV}$, a value which is roughly $2 \mathrm{eV}$ greater than the lowest energy excitation rationalizing, in line with the argument above, the experimentally determined lack of visible light charge separation in the solid film, Table S2.

In several recent studies employing supramolecular PBIs as photoconductors and photocatalysts, additional external electron donors (e.g., methanol, diethylamine) have led to visible light activity. ${ }^{2,37,38}$ We therefore decided to explore the effect of methanol vapor on the current-voltage response of dried PBI-A films under visible light illumination, Figure $1 \mathrm{~b}$. Methanol vapor was employed here as it offers the opportunity to introduce an alternative electron donor $\left(E_{\mathrm{OX}} \sim-0.4 \mathrm{~V}_{\mathrm{Ag} / \mathrm{AgCl}}\right.$ at $\mathrm{pH} 9)^{39}$ directly into the solid self-assembled PBI-A film. In the presence of the methanol donor, we observe a large decrease in resistance under visible light illumination $(523 \mathrm{~nm}$, Figure $1 b$ ) demonstrating that excitation of the $S_{0}-S_{1}$ transition of the dried PBI-A film can induce the sample to become conductive. The TA spectrum of a dried PBI-A film in the presence of methanol vapor confirms that it is indeed possible to form a $\mathrm{PBI}^{\bullet-}$-A radical following $490 \mathrm{~nm}$ excitation, with the bands of the reduced PBI at 545, 735, and 812 being present within 1 ps and persisting for $>3.2 \mathrm{~ns}$, Figures $4 \mathrm{c}$, d. It is therefore apparent that by careful control of the inclusion of a suitable electron donor to the PBI-A that visible light driven charge separation can be enabled, in which the exciton oxidizes methanol, highlighting the feasibility of developing efficient donor-acceptor heterojunctions based on self-assembled amino acid appended PBI acceptors.

\section{CONCLUSIONS}

Self-assembled structures formed from amino acid PBIs are known to be highly promising photoconductive materials due to their reported oxygen tolerance and ability to form longrange one-dimensional structures both in dried solutions and when gelled. However, a significant issue has been multiple amino acid PBIs have reported photoactivity primarily under UV illumination, despite the UV/vis absorption spectrum extending into the visible region. ${ }^{6,40,41}$ Here, we use TA spectroscopy to show that $\mathrm{PBI}^{\circ-}$, whose presence is known to correlate to the material showing photoconductivity, can be generated within a PBI-A supramolecular structure in water within 1 ps under both $\mathrm{UV}\left(\mathrm{S}_{0}-\mathrm{S}_{2}\right)$ and visible $\left(\mathrm{S}_{0}-\mathrm{S}_{1}\right)$ light excitation. In contrast, when dried films are employed charge separation only occurs under UV illumination due to a destabilization of the charge-separated state in the low dielectric environment and a change in the site of hole localization. In these experiments, it is proposed that the alanine functionality is acting as an electron donor, and this is supported by the observation that the inclusion of a more readily oxidized electron donor (methanol) leads to the switching on of photoactivity under visible light. The differing spectral response of the PBI-A 1-D structures in different dielectric environments represents an important design consideration for the development of these promising materials for use in optoelectronic devices.

\section{ASSOCIATED CONTENT}

\section{Supporting Information}

The Supporting Information is available free of charge on the ACS Publications website at DOI: 10.1021/acs.jpcc.6b06222.

Full description of the experimental methods; variable concentration UV/vis and emission spectroscopy experiments; the calculation of the donor-acceptor distance within an aggregated PBI-A sample; SANS data, kinetic fits, and supplementary DFT information (PDF)

\section{AUTHOR INFORMATION}

\section{Corresponding Authors}

*E-mail: d.j.adams@liverpool.ac.uk.

*E-mail: a.j.cowan@liverpool.ac.uk.

\section{Author Contributions}

The manuscript was written through contributions of all authors. All authors have given approval to the final version of the manuscript.

Notes

The authors declare no competing financial interest.

\section{ACKNOWLEDGMENTS}

J.J.W. and E.R.D. acknowledge the EPSRC for financial support. J.R.L. acknowledges the School of Physics (University of Liverpool) for a GTA studentship. A.J.C., D.J.A., and M.A.Z. thank the EPSRC for fellowships (EP/K006851/1, EP/ L021978/1, and EP/I004424/1 respectively). The Laser Loan Pool of the Central Laser Facility (CLF), part of the UK Science and Technology Facilities Council (STFC), is thanked for the loan of equipment through EP/G03088X/1. Experiments at the ISIS Pulsed Neutron and Muon Source were supported by a beam-time allocation from the STFC (Computational time on ARCHER, the U.K.'s national highperformance computing service (via our membership of the U..K's HPC Materials Chemistry Consortium, which is funded by EPSRC grant EP/L000202/1), and the EPSRC National Service for Computational Chemistry Software is gratefully acknowledged. This work benefitted from the SasView software, originally developed by the DANSE project under NSF award DMR-0520547.

\section{REFERENCES}

(1) Neuteboom, E. E.; Meskers, S. C. J.; Van Hal, P. A.; Van Duren, J. K. J.; Meijer, E. W.; Janssen, R. A. J.; Dupin, H.; Pourtois, G.; Cornil, J.; Lazzaroni, R.; et al. Alternating Oligo(p-phenylene vinylene)Perylene Bisimide Copolymers: Synthesis, Photophysics, and Photovoltaic Properties of a New Class of Donor-Acceptor Materials. J. Am. Chem. Soc. 2003, 125, 8625-8638.

(2) Chen, S.; Jacobs, D. L.; Xu, J.; Li, Y.; Wang, C.; Zang, L. 1D Nanofiber Composites of Perylene Diimides for Visible-Light-Driven Hydrogen Evolution from Water. RSC Adv. 2014, 4, 48486-48491.

(3) Würthner, F.; Saha-Möller, C. R.; Fimmel, B.; Ogi, S.; Leowanawat, P.; Schmidt, D. Perylene Bisimide Dye Assemblies as Archetype Functional Supramolecular Materials. Chem. Rev. 2016, 116, 962-1052. 
(4) Marty, R.; Nigon, R.; Leite, D.; Frauenrath, H. Two-Fold OddEven Effect in Self-Assembled Nanowires from Oligopeptide-PolymerSubstituted Perylene Bisimides. J. Am. Chem. Soc. 2014, 136, 39193927.

(5) Roy, S.; Maiti, D. K.; Panigrahi, S.; Basak, D.; Banerjee, A. A New Hydrogel from an Amino Acid-Based Perylene Bisimide and Its Semiconducting, Photo-Switching Behaviour. RSC Adv. 2012, 2, $11053-11060$.

(6) Draper, E. R; Walsh, J. J.; Mcdonald, T. O.; Zwijnenburg, M. A.; Cameron, P. J.; Cowan, A. J.; Adams, D. J. Air-Stable Photoconductive Films Formed from Perylene Bisimide Gelators. J. Mater. Chem. C 2014, 2, 5570-5575.

(7) Marciniak, H.; Li, X. Q.; Würthner, F.; Lochbrunner, S. OneDimensional Exciton Diffusion in Perylene Bisimide Aggregates. J. Phys. Chem. A 2011, 115, 648-654.

(8) Reilly, T. H.; Hains, A. W.; Chen, H.-Y.; Gregg, B. A. A SelfDoping, $\mathrm{O}_{2}$-Stable, $\mathrm{n}$-Type Interfacial Layer for Organic Electronics. Adv. Energy Mater. 2012, 2, 455-460.

(9) Wicklein, A.; Ghosh, S.; Sommer, M.; Würthner, F.; Thelakkat, M. Self-Assembly of Semiconductor Organogelator Nanowires for Photoinduced Charge Separation. ACS Nano 2009, 3, 1107-1114.

(10) Ye, T.; Singh, R.; Butt, H.; Floudas, G.; Keivanidis, P. E. Effect of Local and Global Structural Order on the Performance of Perylene Diimide Excimeric Solar Cells. ACS Appl. Mater. Interfaces 2013, 5, 11844-11857.

(11) Ley, D.; Guzman, C. X.; Adolfsson, K. H.; Scott, A. M.; Braunschweig, A. B. Cooperatively Assembling Donor-Acceptor Superstructures Direct Energy into an Emergent Charge Separated State. J. Am. Chem. Soc. 2014, 136, 7809-7812.

(12) Hartnett, P. E.; Dyar, S. M.; Margulies, E. A.; Shoer, L. E.; Cook, A. W.; Eaton, S. W.; Marks, T. J.; Wasielewski, M. R. Long-Lived Charge Carrier Generation In Ordered Films of a Covalent Perylenediimide-Diketopyrrolopyrrole-Perylenediimide Molecule. Chem. Sci. 2015, 6, 402-411.

(13) Lim, J. M.; Kim, P.; Yoon, M. C.; Sung, J.; Dehm, V.; Chen, J.; Würthner, F.; Kim, D. Exciton Delocalization and Dynamics in Helical $\Pi$-Stacks of Self-Assembled Perylene Bisimides. Chem. Sci. 2013, 4, 388-397.

(14) Supur, M.; Fukuzumi, S. Photodriven Electron Transport within the Columnar Perylenediimide Nanostructures Self-Assembled with Sulfonated Porphyrins in Water. J. Phys. Chem. C 2012, 116, 2327423282.

(15) Lefler, K. M.; Co, D. T.; Wasielewski, M. R. Self-AssemblyInduced Ultrafast Photodriven Charge Separation in Perylene-3,4dicarboximide-Based Hydrogen-Bonded Foldamers. J. Phys. Chem. Lett. 2012, 3, 3798-3805.

(16) Rybtchinski, B.; Sinks, L. E.; Wasielewski, M. R. Photoinduced Electron Transfer in Self-Assembled Dimers of 3-Fold Symmetric Donor-Acceptor Molecules Based on Perylene-3,4:9,10-bis(dicarboximide). J. Phys. Chem. A 2004, 108, 7497-7505.

(17) Forster, M.; Potter, R. J.; Ling, Y.; Yang, Y.; Klug, D. R; Li, Y.; Cowan, A. J. Oxygen Deficient A-Fe $\mathrm{O}_{2} \mathrm{O}_{3}$ Photoelectrodes: A Balance between Enhanced Electrical Properties and Trap-Mediated Losses. Chem. Sci. 2015, 6, 4009-4016.

(18) Stephens, P. J.; Devlin, F. J.; Chabalowski, C. F.; Frisch, M. J. Ab Initio Calculation of Vibrational Absorption and Circular Dichroism Spectra Using Density Functional Force Fields. J. Phys. Chem. 1994, 98, 11623-11627.

(19) Becke, A. D. Density-Functional Thermochemistry. III. The Role Of Exact Exchange. J. Chem. Phys. 1993, 98, 5648.

(20) Grimme, S.; Antony, J.; Ehrlich, S.; Krieg, H. A Consistent And Accurate $A b$ Initio Parametrization of Density Functional Dispersion Correction (DFT-D) for the 94 Elements H-Pu. J. Chem. Phys. 2010, $132,154104$.

(21) Schäfer, A.; Horn, H.; Ahlrichs, R. Fully Optimized Contracted Gaussian Basis Sets for Atoms Li to Kr. J. Chem. Phys. 1992, 97, 25712577.

(22) Weigend, F.; Ahlrichs, R. Balanced Basis Sets of Split Valence, Triple Zeta Valence and Quadruple Zeta Valence Quality for H to Rn:
Design and Assessment of Accuracy. Phys. Chem. Chem. Phys. 2005, 7, 3297-3305.

(23) Klamt, A.; Schüürmann, G. COSMO: A New Approach to Dielectric Screening in Solvents with Explicit Expressions for the Screening Energy and Its Gradient. J. Chem. Soc., Perkin Trans. 2 1993, 5, 799-805.

(24) Ahlrichs, R.; Bär, M.; Häser, M.; Horn, H.; Kölmel, C. Electronic Structure Calculations on Workstation Computers: The Program System Turbomole. Chem. Phys. Lett. 1989, 162, 165-169.

(25) Furche, F.; Ahlrichs, R.; Hättig, C.; Klopper, W.; Sierka, M.; Weigend, F. Turbomole. WIREs Comput. Mol. Sci. 2014, 4, 91-100.

(26) Ambrosek, D.; Marciniak, H.; Lochbrunner, S.; Tatchen, J.; Li, X.-Q.; Würthner, F.; Kühn, O. Photophysical and Quantum Chemical Study on a J-Aggregate Forming Perylene Bisimide Monomer. Phys. Chem. Chem. Phys. 2011, 13, 17649-17657.

(27) Raeburn, J.; Mendoza-Cuenca, C.; Cattoz, B. N.; Little, M. A.; Terry, A. E.; Cardoso, A. Z.; Griffiths, P. C.; Adams, D. J. The Effect of Solvent Choice on the Gelation and Final Hydrogel Properties of Fmoc-Diphenylalanine. Soft Matter 2015, 11, 927-935.

(28) Draper, E. R.; Mears, L. L. E.; Castilla, A. M.; King, S. M.; McDonald, T. O.; Akhtar, R.; Adams, D. J. Using the Hydrolysis of Anhydrides To Control Gel Properties And Homogeneity in pHTriggered Gelation. RSC Adv. 2015, 5, 95369-95378.

(29) van der Boom, T.; Hayes, R. T.; Zhao, Y.; Bushard, P. J.; Weiss, E. A.; Wasielewski, M. R. Charge Transport in Photofunctional Nanoparticles Self-Assembled from Zinc 5,10,15,20-Tetrakis(perylenediimide)porphyrin Building Blocks. J. Am. Chem. Soc. 2002, $124,9582-9590$.

(30) Alhedabi, T.; Herlem, G.; Cattey, H.; Blondeau-Patissier, V.; Gharbi, T. Electrosynthesis of Poly(alanine)-Like Peptides in Concentrated Alanine Based Electrolytes, Characterization Coupled to DFT Study and Application to $\mathrm{pH}$ Proton Receptor. J. Phys. Chem. C 2014, 118, 25041-25050.

(31) Ronconi, F.; Syrgiannis, Z.; Bonasera, A.; Prato, M.; Argazzi, R.; Caramori, S.; Cristino, V.; Bignozzi, C. A. Modification of Nanocrystalline $\mathrm{WO}_{3}$ with a Dicationic Perylene Bisimide: Applications to Molecular Level Solar Water Splitting. J. Am. Chem. Soc. 2015, 137, $4630-4633$

(32) Lindquist, R. J.; Phelan, B. T.; Reynal, A.; Margulies, E. A.; Shoer, L. E.; Durrant, J. R.; Wasielewski, M. R. Strongly Oxidizing Perylene-3,4-Dicarboximides for Use in Water Oxidation Photoelectrochemical Cells. J. Mater. Chem. A 2016, 4, 2880-2893.

(33) Würthner, F.; Sautter, A.; Schmid, D.; Weber, P. J. A. Fluorescent and Electroactive Cyclic Assemblies from Perylene Tetracarboxylic Acid Bisimide Ligands and Metal Phosphane Triflates. Chem.-Eur. J. 2001, 7, 894-902.

(34) Natarajan, S.; Britto, S. A. M.; Ramachandran, E. Growth, Thermal, Spectroscopic, and Optical Studies of 1-Alaninium Maleate, a New Organic Nonlinear Optical Material. Cryst. Growth Des. 2006, 6, 137-140.

(35) Wu, Y.; Young, R. M.; Frasconi, M.; Schneebeli, S. T.; Spenst, P.; Gardner, D. M.; Brown, K. E.; Würthner, F.; Stoddart, J. F.; Wasielewski, M. R. Ultrafast Photoinduced Symmetry-Breaking Charge Separation and Electron Sharing in Perylenediimide Molecular Triangles. J. Am. Chem. Soc. 2015, 137, 13236-13239.

(36) Nowak-Król, A.; Fimmel, B.; Son, M.; Kim, D.; Würthner, F. Photoinduced Electron Transfer (PET) Versus Excimer Formation in Supramolecular P/N-Heterojunctions of Perylene Bisimide Dyes and Implications for Organic Photovoltaics. Faraday Discuss. 2015, 185, 507-527.

(37) Datar, A.; Balakrishnan, K.; Zang, L. One-Dimensional SelfAssembly of a Water Soluble Perylene Diimide Molecule by $\mathrm{Ph}$ Triggered Hydrogelation. Chem. Commun. 2013, 49, 6894-6896.

(38) Weingarten, A. S.; Kazantsev, R. V.; Palmer, L. C.; McClendon, M.; Koltonow, A. R.; Samuel, A. P. S.; Kiebala, D. J.; Wasielewski, M. R; Stupp, S. I. Self-Assembling Hydrogel Scaffolds for Photocatalytic Hydrogen Production. Nat. Chem. 2014, 6, 964-970.

(39) Berr, M. J.; Wagner, P.; Fischbach, S.; Vaneski, A.; Schneider, J.; Susha, A. S.; Rogach, A. L.; Jäckel, F.; Feldmann, J. Hole Scavenger 
Redox Potentials Determine Quantum Efficiency and Stability of PtDecorated Cds Nanorods for Photocatalytic Hydrogen Generation. Appl. Phys. Lett. 2012, 100, 223903.

(40) Chen, S.; Li, Y.; Wang, C. Visible-Light-Driven Photocatalytic $\mathrm{H}_{2}$ Evolution from Aqueous Suspensions of Perylene Diimide DyeSensitized Pt/ $\mathrm{TiO}_{2}$ Catalysts. RSC Adv. 2015, 5, 15880-15885.

(41) Chen, S.; Wang, C.; Bunes, B. R.; Li, Y.; Wang, C.; Zang, L. Enhancement Of Visible-Light-Driven Photocatalytic $\mathrm{H}_{2}$ Evolution from Water Over $\mathrm{g}-\mathrm{C}_{3} \mathrm{~N}_{4}$ through Combination with Perylene Diimide Aggregates. Appl. Catal., A 2015, 498, 63-68.

\section{NOTE ADDED AFTER ASAP PUBLICATION}

This paper was published to the Web on August 12, 2016, with an error in the TOC/abstract graphics. This was corrected in the version published to the Web on August 17, 2016. 